\title{
A non-homogeneous discrete time Markov model for admission scheduling and resource planning in a cost or capacity constrained healthcare system
}

\author{
Lalit Garg • Sally McClean • Brian Meenan • \\ Peter Millard
}

Received: 5 March 2009/Accepted: 23 October 2009

(C) Springer Science+Business Media, LLC 2009

\begin{abstract}
Healthcare resource planners need to develop policies that ensure optimal allocation of scarce healthcare resources. This goal can be achieved by forecasting daily resource requirements for a given admission policy. If resources are limited, admission should be scheduled according to the resource availability. Such resource availability or demand can change with time. We here model patient flow through the care system as a discrete time Markov chain. In order to have a more realistic representation, a nonhomogeneous model is developed which incorporates timedependent covariates, namely a patient's present age and the present calendar year. The model presented in this paper can be used for admission scheduling, resource requirement forecasting and resource allocation, so as to satisfy the demand or resource constraints or to meet the expansion or contraction plans in a hospital and community based integrated care system. Such a model can be used with both fixed and variable numbers of admissions per day and should prove to be a useful tool for care managers and policy makers who require to make strategic management decisions. We also describe an application of the model to an elderly care system,
\end{abstract}

L. Garg $(\bowtie) \cdot$ S. McClean

School of Computing and Information Engineering,

University of Ulster,

Cromore Road,

Coleraine, BT54 1SA Northern Ireland, UK

e-mail: lg1in@yahoo.com

B. Meenan

School of Electrical and Mechanical Engineering,

University of Ulster,

Jordanstown, Northern Ireland, UK

P. Millard

St George's, University of London,

London, UK using a historical dataset from the geriatric department of a London hospital.

Keywords Resource management · Admission scheduling · Non-homogeneous Markov model · Stochastic optimal control

\section{Introduction}

Admission scheduling [1, 2] and resource planning [3] are fundamental problems which require complex strategies to effectively manage care services ensuring optimum utilization of scarce resources and efficient quality of service delivery. Long waiting lists are considered to be symptomatic of an inefficient care system [4-6]. To avoid long waiting lists, care professionals and policy makers are required to estimate the resource requirements for a given time in the future and to allocate the budget/ resources to satisfy this demand [7]. Also if the resource availability is limited and cannot meet an ever increasing demand, it is necessary to estimate the number of admissions that can be satisfied each day and schedule accordingly, from the waiting list [8]. This will help the health care managers to optimally exploit capacity and limit the size of the waiting list [7].

Neither admission scheduling nor resource requirements forecasting are new problems and numerous models have been proposed. Some of these [5, 9-13] are queueing models, others [14-19] utilise simulation or population/ ratio based approaches which use average lengths of stay to quantify the number of patients requiring care resources, for example [20-24]. However, some of these methods, which are mostly stochastic or simulation based, are computationally very complex and require substantial data and logistics [25]. Other simpler methods, on the other hand, do not properly represent case mix [3] or local specificities [25] 
and thus require more sophisticated models [26]. In addition, as suggested by [3, 27], other approaches are inaccurate or misleading. Also previous models mainly model patient flow in a department or hospital and do not incorporate readmissions and care in the community. Therefore they are not very suitable for admission scheduling and capacity planning in an integrated care system, which includes both hospital and community care [28].

In our previous work [29] we have developed a continuous time non-homogeneous Markov model for admission scheduling and resource forecasting by enumerating patient pathways. The limitation of this model is that it assumes a fixed number of admissions per day, so it cannot be used in many practical scenarios (such as a care system with expansion or contraction plans). A variable number of admissions each day can therefore prove to be a more realistic assumption with correspondingly better solutions that ensure optimal resource utilization. Also we might need to schedule a variable number of admissions each day so as to allocate resources to satisfy the fluctuating demand for care services [12, 30]. In this paper, we present a discrete time Markov model for admission scheduling and capacity planning. Based on our initial homogeneous model, a novel, more realistic nonhomogeneous model is developed, incorporating time dependent covariates (in this case the patient's present age and the present calendar year). This approach can effectively be used for resource requirement forecasting and resource allocation to satisfy the demand or resource constraints or to meet the expansion or contraction plans. We can also use this new model to compare different admission scheduling strategies for a care system. Finally we illustrate use of the model by applying it to a historical dataset of all male patients from a geriatric department of a London hospital admitted during a 16 year period [31]. Throughout the paper we use mathematical notation which is described in Appendix 2 (Table 5).

\section{Background}

\subsection{Patient flow through the care system}

An integrated care system is a system in which patient care is provided in both hospital and the community. Patient flow can be characterized by the rate of transition of a patient from one phase to another in this care system. A cohort analysis of patient flows through all phases in a care system can help us to forecast the number of patients in various phases at a given time in the future [32]. In most cases patient flow depends on the patient pathways defined for the care system. We assume that a care system has $n$ hospital phases, such as acute, treatment, rehabilitation, or long stay and $m$ community phases, such as dependent, convalescent, or recovered. These partially observable phases can be real such as various hospital or community care units or conceptual, representing periods in hospital and/or community care depending on the patient phase of care or stage of the illness being treated $[33,34]$. Figure 1 is the schematic representation of patient flow through the care system.

A patient pathway for such a system is defined as the way a patient sequentially moves from one hospital phase to the next and similarly one community phase to the next. Patients can be discharged from any hospital phase to the first community phase or the patient can die in any hospital or community phase. Re-admission into the first hospital phase is possible from any community phase. We can therefore represent this care system by an $n+m+1$ state discrete time Markov chain with death as an absorbing state. The transition matrix $\mathbf{Q}$ for the absorbing Markov chain can then be represented as follows [33, 35]:

$\mathbf{Q}=\left\{q_{i j}\right\}=$ Transition rate (next transition is to phase $j \mid$ currently in phase $i$ ).

$$
\mathbf{Q}=\left(\begin{array}{ccccc:ccccc:c}
-\lambda_{1}-\mu_{1}-v_{1} & \lambda_{1} & 0 & \ldots & 0 & v_{1} & 0 & 0 & \ldots & 0 & \mu_{1} \\
0 & -\lambda_{2}-\mu_{2}-v_{2} & \lambda_{2} & \ldots & 0 & v_{2} & 0 & 0 & \ldots & 0 & \mu_{2} \\
. & . & . & \ldots & . & . & . & . & \ldots & . & \cdot \\
. & . & . & \ldots & \lambda_{n-1} & . & . & . & \ldots & . & . \\
0 & 0 & . & \ldots & -\mu_{n}-v_{n} & v_{n} & 0 & 0 & \ldots & \mu_{n} \\
\hdashline \gamma_{1} & 0 & 0 & \ldots & 0 & -\alpha_{1}-\beta_{1}-\gamma_{1} & \alpha_{1} & 0 & \ldots & 0 & \beta_{1} \\
\gamma_{2} & 0 & 0 & \ldots & 0 & 0 & -\alpha_{2}-\beta_{2}-\gamma_{2} & \alpha_{2} & \ldots & 0 & \beta_{2} \\
. & . & . & \ldots & . & . & . & . & \ldots & . & . \\
. & . & . & \ldots & . & . & . & . & \ldots & . & . \\
\hdashline \gamma_{m} & 0 & 0 & \ldots & 0 & 0 & 0 & 0 & \ldots & -\beta_{m}-\gamma_{m} & \beta_{m} \\
\hdashline 0 & 0 & 0 & \ldots & 0 & 0 & 0 & 0 & \ldots & 0 & 0
\end{array}\right) .
$$


Fig. 1 Patient flow in the care system. Here HP $i$ represents hospital phase $i$ and $\mathrm{CP} i$ represents community phase $i$

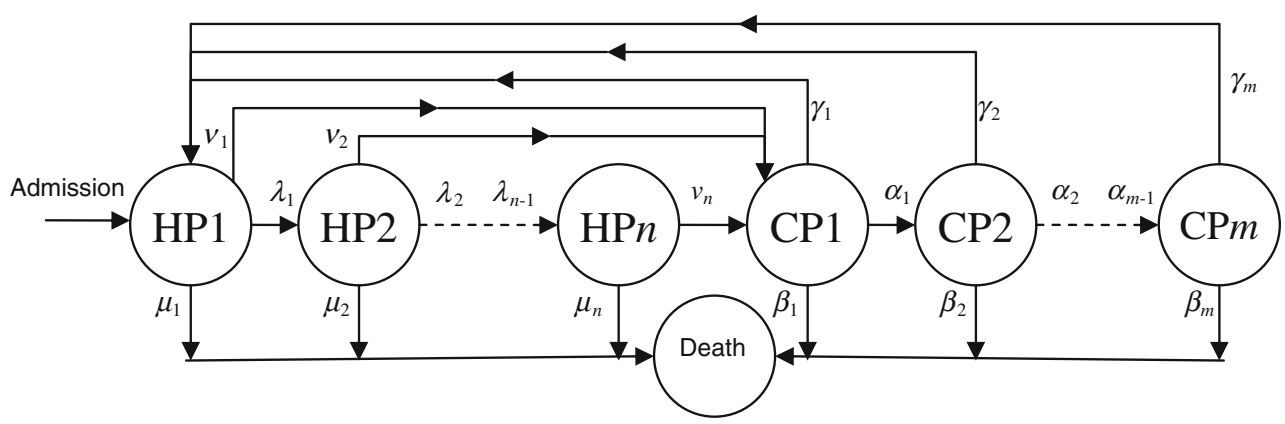

Here $\lambda_{i}$ represents the rate of transition from hospital phase $i$ to hospital phase $i+1 . \nu_{i}$ represents the rate of transition from hospital phase $i$ to the first community phase and $\mu_{i}$ is the rate of transition from hospital phase $i$ to the absorbing phase death. Similarly $\alpha_{i}$ is the rate of transition from community phase $i$ to community phase $i+$ $1, \gamma_{i}$ is the rate of transition from community phase $i$ to the first hospital phase and $\beta_{i}$ is the rate of transition from community phase $i$ to death.

We assume that the duration $t$ of a step is 1 day. Therefore, the discrete time transition probability matrix can be defined as $\mathbf{P}=\left\{p_{i j}\right\}=$ Probability (next transition is to phase $j \mid$ currently in phase $i$ ), where $\mathbf{P}$ can be described as follows [35]:

$\mathbf{P}=\exp (\mathbf{Q} t)=\exp (\mathbf{Q})$.

\section{Model description}

\subsection{The homogeneous model}

In the previous section we have discussed how we can model a care system as a discrete time absorbing Markov chain, based on our continuous time representation. The vector $\mathbf{s}_{\mathbf{0}}$ represents the initial values of number of patients in different phases of the system.

$\mathbf{s}_{0}=\left\{s_{0,1}, s_{0,2}, s_{0,3}, \ldots, s_{0, n}, s_{0, n+1}, s_{0, n+2}, \ldots, s_{0, n+m}, s_{0, n+m+1}\right\}$.

The mean number of patients in each phase after $k$ days is given by vector $\mathbf{s}_{k}$, where:

$\mathbf{s}_{k}=\mathbf{s}_{0} * \mathbf{P}^{k}$.

We also define a cost vector $\mathbf{c}=\left\{c_{\mathrm{i}}\right\}=$ daily cost of care in phase $i$ :

$\mathbf{c}=\left(c_{1}, c_{2}, c_{3}, \ldots, c_{n+m}, 0\right)^{\prime}$

where $c_{n+m+1}=0$ (there is no cost in the death phase).

\subsection{The non-homogeneous model}

Typically patient flow also depends on time dependent covariates such as the patient's age and the calendar year. In order to have a more realistic model it is therefore necessary to update parameter values with time [35]. The transition rates $\lambda_{i}, \mu_{i}, \nu_{i}, \alpha_{i}, \beta_{i}$ and $\gamma_{i}$ have been previously assumed to depend log-linearly on the time dependent covariates $\chi=\left(\begin{array}{lllll}\chi_{1} & \chi_{2} & \ldots & \chi_{n}\end{array}\right)$. The log-linear function here takes the form:

$\exp \left(\sigma+\mathbf{b}^{\prime} \chi\right)$

where coefficient parameters $\sigma$ and vector $\mathbf{b}$ are estimated for each of the transition rates using maximum likelihood estimation [33, 35].

In the model presented here we consider two time dependent covariates: the patient's present age and the present calendar year. In order to have a more realistic model, we update the covariates each day for each patient currently in the system, recalculate the parameter values for each patient separately and recompute the discrete time transition probability matrix $\mathbf{P}$.

The mean number of patients in each phase after $k$ days is then given by:

$\mathbf{s}_{k}=\mathbf{s}_{0} * \prod_{i=1}^{k} \mathbf{P}^{(i)}$

where $\mathbf{P}^{(i)}$ represents the value of transition matrix $\mathbf{P}$ on day $i$.

The estimated number of patients in the care system on day $k$ is:

$\eta_{k}=\sum_{i=1}^{n+m} s_{k, i}=\mathbf{s}_{k} *(\mathbf{h}+\mathbf{e})$

where $\mathbf{h}$ is a column vector of $(n+m+1)$ elements. Here the first $n$ elements (number of hospital phases) are 1 and the remaining elements are 0 , and $\mathbf{e}$ is a column vector of $(n+m+$ 1) elements, with first $n$ elements and last element equal to 0 and the remaining $m$ elements (number of community phases) are 1 . The column vectors $\mathbf{h}$ and $\mathbf{e}$ are used to separately estimate the expected number of patients in hospital and community phases.

The expected number of patients in the hospital after $k$ days is:

$\eta_{k}^{h}=\mathbf{s}_{k} * \mathbf{h}$. 
The expected number of patients in the community after $k$ days is:

$\eta_{k}{ }^{c}=\mathbf{s}_{k}{ }^{*} \mathbf{e}$

and the expected total daily cost on day $k$ is

$\Omega_{k}=\mathbf{s}_{k} * \mathbf{c}$.

Once the expected numbers of patients in different phases of the system are known, we use these expressions to calculate the optimum rate of admissions to satisfy the given constraints and to estimate the resource requirements based on the given admission schedule.

\subsection{Incorporating admissions}

For a more realistic model it is necessary to incorporate new admissions into the system. We therefore develop our model to include, in the first instance, a fixed number of admissions each day and subsequently a variable number of admissions (increasing or decreasing) each day are assumed.

\subsubsection{Fixed number of admissions}

For modelling a fixed number of admissions each day, we first assume that initially there are no patients in the system and on day 0 the first patient is admitted into the first hospital phase. On each subsequent day, one new patient is admitted into the first hospital phase. We will separately estimate the distribution vector for each patient and sum all these to estimate the patients' distribution on a given day. Here the individual age of each patient is considered. Thus the vector of updated values of patients in different phases after $k$ days is given by:

$\mathbf{s}_{k}=\mathbf{s}_{0} * \sum_{j=1}^{k}\left(\prod_{i=1}^{k-j+1} \mathbf{P}_{j}^{(i)}\right)$

where $\mathbf{s}_{0}=\{1,0,0,0, \ldots \ldots, 0, \ldots 0\}$ representing the fact that on day 0 the first patient is admitted into the first hospital phase and $\mathbf{P}^{(i)}{ }_{j}$ is the transition probability matrix for patient $j$ on day $i$. Here $\mathbf{s}_{k}$ represents the number of patients in different phases after $k$ days with 1 admission per day. If the admission rate is $a$ admissions per day, then the expected number of patients in different phases after $k$ days will be $a^{*} \mathbf{s}_{k}$.

\subsubsection{Variable number of admissions}

For a care system having a variable number of admissions each day we assume that the rate of change in admission rate (growth in service) is $r$. Here $r$ can be a positive (growth) or negative (contraction) real number. Therefore the expected number of patients in different phases on day $k$ will be:

$\mathbf{s}_{k}=\mathbf{x}+\mathbf{y}$

where $\mathbf{x}$ is the vector of expected number of patients after $k$ days with a fixed number of admissions each day and $\mathbf{y}$ is the vector of the change in the expected number of patients in each phase after $k$ days due to the change in admission rate. Therefore

$$
\begin{aligned}
\mathbf{y} & =\mathbf{s}_{0} * \sum_{j=1}^{k}\left(\left(r^{*} j\right)^{*} \prod_{i=1}^{k-j+1} \mathbf{P}_{j}^{(i)}\right) \\
& =r^{*} \mathbf{s}_{0} *\left(\sum_{j=1}^{k}\left(j^{*} \prod_{i=1}^{k-j+1} \mathbf{P}_{j}^{(i)}\right)\right) .
\end{aligned}
$$

Thus the patient distribution on day $k$ will be:

$\mathbf{s}_{k}=\mathbf{s}_{0} *\left\{\sum_{j=1}^{k}\left(\prod_{i=1}^{k-j+1} \mathbf{P}_{j}^{(i)}\right)+r^{*}\left(\sum_{j=1}^{k}\left(j^{*} \prod_{i=1}^{k-j+1} \mathbf{P}_{j}^{(i)}\right)\right)\right\}$.

So we can write:

$\mathbf{s}_{k}=\mathbf{x}+r^{*} \mathbf{w}$.

where vector $\mathbf{w}$ represents the expected change in patient distribution (number of patients in different phases) due to unit change (1 patient per day) in admission rate. Similarly we can have more complex functions for admission rate comprising higher powers of $\mathbf{w}$ such as

$\mathbf{s}_{k}==\mathbf{x}+r_{1}{ }^{*} \mathbf{w}_{1}+r_{2}{ }^{*} \mathbf{w}_{2}+r_{3}{ }^{*} \mathbf{w}_{3}+\ldots \ldots . .+r_{n}{ }^{*} \mathbf{w}_{n}$

where $\mathbf{w}_{m}=\sum_{j=1}^{k}\left(j^{m *} \prod_{i=1}^{k-j+1} \mathbf{P}_{j}^{(i)}\right)$.

The results obtained in this sub-section can be used for admission scheduling as discussed in the next sub-section.

\subsection{Admission scheduling}

Admission scheduling can be defined as designing an admission policy to ensure optimum utilization of the future available resources satisfying given constraints or meeting a given target. Possible constraints can be availability based on budgets, beds or other resources (such as nursing staff, physicians, or specialists) at a given time in the future or the total daily, monthly or yearly expenditure. We will consider two scenarios of interest: first a new care system and second a pre-existing care system. 


\subsubsection{A new care system}

In a new care system the first patient is admitted into hospital phase 1 on day 0 (at $t=0$ ). Therefore for a new care system:

$\mathbf{s}_{0}=\{1,0,0,0, \ldots \ldots, 0, \ldots 0\}$.

We assume that the constraint is to satisfy the available number of beds, so there will be only $B\left(t_{\text {given }}\right)$ beds available at time $t_{\text {given }}$.

Therefore the expected number of admissions allowed each day can be calculated as:

$A_{\text {req }}=\frac{B\left(t_{\text {given }}\right)}{\eta_{t_{\text {given }}}^{h}}$ per day

where, $\eta_{t_{\text {given }}}$ is the expected number of patients in the care system at time $t_{\text {given }}$ and is defined in (5).

If the constraint is the total number of beds available in a hospital but the beds availability in the community is not constrained, then the expected number of admissions is:

$A_{\text {req }}=\frac{C\left(t_{\text {given }}\right)}{\Omega_{t_{\text {given }}}}$ per day

where $\eta_{t_{\text {given }}}^{h}$ is the number of patients in hospital at time $t_{\text {given }}$ and is defined in (6).

If the total daily cost of care is a constraint then the expected number of admissions is:

$A_{\text {req }}=\frac{C\left(t_{\text {given }}\right)}{\Omega_{t_{\text {given }}}}$ per day

where $C\left(t_{\text {given }}\right)$ is the available daily budget (cost) at time $t_{\text {given. }} \Omega_{t_{\text {given }}}$ is the expected total daily cost at time $t_{\text {given }}$ and is defined in (8).

Here it is important to note that we are required to define the rate of change in admission rate $(r)$ so as to calculate the number of admissions allowed each day. For a system with a fixed number of admissions each day, $r=0$. We can also calculate the value of $r$ if the resource constraints are available for two different dates $\left(t_{1}\right.$ and $\left.t_{2}\right)$ in the future. For example if the total daily cost constraint is given for two dates in the future, with $C\left(t_{1}\right)$ the cost at time $t_{1}$ and $C\left(t_{2}\right)$ the cost at time $t_{2}$, from (8) and (13):

$\Omega_{t_{1}}=\left(\mathbf{x}_{1}+\mathrm{r}^{*} \mathbf{w}_{1}\right) * \mathbf{c}$

and similarly

$\Omega_{t_{2}}=\left(\mathbf{x}_{2}+\mathrm{r}^{*} \mathbf{w}_{2}\right) * \mathbf{c}$.

As the initial rates of admission should be the same, using (17) we get:

$\frac{C\left(t_{1}\right)}{\Omega_{t_{1}}}=\frac{C\left(t_{2}\right)}{\Omega_{t_{2}}}$ and this gives:

$r=\left(\frac{\left(C\left(t_{1}\right) *\left(\mathbf{x}_{2} * \mathbf{c}\right)\right)-\left(C\left(t_{2}\right) *\left(\mathbf{x}_{1} * \mathbf{c}\right)\right)}{\left(C\left(t_{2}\right) *\left(\mathbf{w}_{1} * \mathbf{c}\right)\right)-\left(C\left(t_{1}\right) *\left(\mathbf{w}_{2} * \mathbf{c}\right)\right)}\right)$

From (20) it is clear that the value of $r$ will be negative for a hospital with a contraction plan.

If we want to keep the total cost constant for a given duration in the future (from $t_{1}$ to $t_{2}$ ):

$C\left(t_{1}\right)=C\left(t_{2}\right)=C(t)$,

so

$r=\left(\frac{\left(\mathbf{x}_{2} * \mathbf{c}\right)-\left(\mathbf{x}_{1} * \mathbf{c}\right)}{\left(\mathbf{w}_{1} * \mathbf{c}\right)-\left(\mathbf{w}_{2} * \mathbf{c}\right)}\right)$.

Similarly, if the bed availability is given for two dates in the future, $\mathrm{B}\left(t_{1}\right)$ at time $t_{1}$ and $\mathrm{B}\left(t_{2}\right)$ at time $t_{2}$, from (5) and (13) we obtain:

$\eta_{t_{1}}=\left(\mathbf{x}_{1}+r^{*} \mathbf{w}_{1}\right) *(\mathbf{h}+\mathbf{e})$,

$\eta_{t_{2}}=\left(\mathbf{x}_{2}+r^{*} \mathbf{w}_{2}\right) *(\mathbf{h}+\mathbf{e})$

and the initial rates of admission should be the same. Using (15) we obtain:

$\frac{B\left(t_{1}\right)}{\eta_{t_{1}}}=\frac{B\left(t_{2}\right)}{\eta_{t_{2}}}$

therefore:

$r=\left(\frac{\left(B\left(t_{1}\right) *\left(\mathbf{x}_{2} *(\mathbf{h}+\mathbf{e})\right)\right)-\left(B\left(t_{2}\right) *\left(\mathbf{x}_{1} *(\mathbf{h}+\mathbf{e})\right)\right)}{\left(B\left(t_{2}\right) *\left(\mathbf{w}_{1} *(\mathbf{h}+\mathbf{e})\right)\right)-\left(B\left(t_{1}\right) *\left(\mathbf{w}_{2} *(\mathbf{h}+\mathbf{e})\right)\right)}\right)$.

If the bed availability remains constant for a given duration in future (from $t_{1}$ to $t_{2}$ ), then:

$B\left(t_{1}\right)=B\left(t_{2}\right)=B(t)$.

Hence:

$r=\left(\frac{\left(\mathbf{x}_{2} *(\mathbf{h}+\mathbf{e})\right)-\left(\mathbf{x}_{1} *(\mathbf{h}+\mathbf{e})\right)}{\left(\mathbf{w}_{1} *(\mathbf{h}+\mathbf{e})\right)-\left(\mathbf{w}_{2} *(\mathbf{h}+\mathbf{e})\right)}\right)$.

\subsubsection{A pre-existing care system}

For a pre-existing care system there are already some patients in various hospital and community phases of the care system at time $t=0$. Therefore, one or more of the elements of $\mathbf{s}_{0}$ is non-zero. For such a care system first we should find the expected number of patients in each phase at time $t=t_{\text {given }}$ days without any new admissions. Then we find the distribution of the new patients. Therefore, the 
distribution of the patients without new admissions (from (4)) is:

$\widetilde{\mathbf{s}}_{t_{\text {given }}}=\widetilde{\mathbf{s}}_{0} * \prod_{i=1}^{t_{\text {given }}} \mathbf{P}^{(i)}$

$\widetilde{\eta}_{t_{\text {given }}}=\widetilde{\mathbf{s}}_{t_{\text {given }}} *(\mathbf{h}+\mathbf{e})=\widetilde{\mathbf{s}}_{0} * \prod_{i=1}^{t_{\text {given }}} \mathbf{P}^{(i)} *(\mathbf{h}+\mathbf{e})$

and the distribution of newly admitted patients (from (5)) is:

$\eta_{t_{\mathrm{given}}}=\mathbf{s}_{t_{\mathrm{given}}} *(\mathbf{h}+\mathbf{e})$

where $\mathbf{s}_{t_{\text {given }}}$ can be calculated from (12) with

$\mathbf{s}_{0}=\{1,0,0,0, \ldots \ldots, 0, \ldots 0\}$.

Thus the expected number of admissions allowed is:

$A_{\text {req }}=\frac{B\left(t_{\text {given }}\right)-\widetilde{\eta}_{t_{\text {given }}}}{\eta_{t_{\text {given }}}}$ per day.

Similarly if the total daily cost of care is a constraint, the expected number of admissions is:

$A_{\text {req }}=\frac{C\left(t_{\text {given }}\right)-\widetilde{\Omega}_{t_{\text {given }}}}{\Omega_{t_{\text {given }}}}$ per day

where $\widetilde{\Omega}_{t_{\text {given }}}=\widetilde{\mathbf{s}}_{t_{\text {given }}} * \mathbf{c}$.

We can use (28) and (29) to estimate the expected admission rate to satisfy the given bed availability constraints and the expected cost of care constraints respectively.

If a care system has variable number of admissions each day the initial rate of admission $a$ is given then from Eq. 13, and the rate of change in admission rate is

$r=\frac{\left(\mathbf{s}_{t_{\text {given }}}-\mathbf{x}\right)}{\mathbf{w}}$.

From (28)

$\mathbf{s}_{t_{\text {given }}}=\frac{B\left(t_{\text {given }}\right)-\widetilde{\eta}_{t_{\text {given }}}}{a^{*}(\mathbf{h}+\mathbf{e})}$.
Therefore the rate of change in admission rate is

$r=\frac{\left(\frac{B\left(t_{\text {given }}\right)-\widetilde{\eta}_{\text {given }}}{a^{*}(\mathbf{h}+\mathbf{e})}-\mathbf{x}\right)}{\mathbf{w}}=\frac{B\left(t_{\text {given }}\right)-\widetilde{\eta}_{t_{\text {given }}}-a^{*} \mathbf{x}^{*}(\mathbf{h}+\mathbf{e})}{a^{*} \mathbf{w}^{*}(\mathbf{h}+\mathbf{e})}$.

Similarly from (29) the rate of change in admission rate is

$r=\frac{\left(\frac{C\left(t_{\text {given }}\right)-\widetilde{\Omega}_{t_{\text {given }}}}{a^{*} \mathbf{c}}-\mathbf{x}\right)}{\mathbf{w}}=\frac{C\left(t_{\text {given }}\right)-\widetilde{\Omega}_{t_{\text {given }}}-a^{*} \mathbf{x}^{*} \mathbf{c}}{a^{*} \mathbf{w}^{*} \mathbf{c}}$.

\section{An illustration}

We illustrate the use of our model using the example of an 8-phase integrated care system proposed by Faddy and McClean [33] comprising of four hospital phases (acute, treatment, rehabilitative and long-stay), three community phases (dependent, convalescent and recovered) and one absorbing phase, death. Faddy and McClean [35] estimated parameters for the Coxian distribution fitting this 8-phase model to the dataset of all male patients admitted between 1969 and 1984 at the geriatric department of a London hospital [31]. In this paper [35] the authors used penalized maximum likelihood estimation with a penalty against multi-model distributions (penalty against distributions where the transition matrix $\mathbf{Q}$ had disparate eigenvalues) thus avoiding over-fitting which might underestimate the effects of time dependent covariates. They used the Nelder Mead algorithm [36] routine of MATLAB software [37] to perform the minimization. Figure 2 is the schematic representation of this care system.

\subsection{Daily resource requirement}

We can estimate the daily resource requirements in a care unit or a care system if we know the expected number of

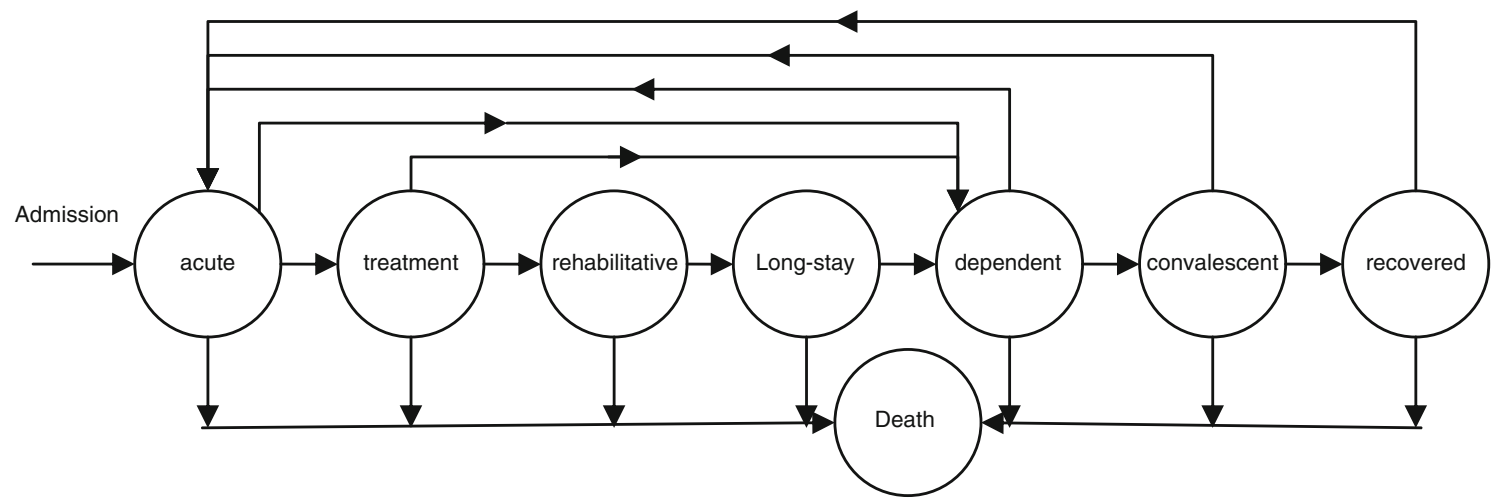

Fig. 2 Patient flow in the eight phase care system 
patients in each phase in the care unit at a given time in future. To calculate the expected cost we have used the indicative cost for the 8-phase care system estimated by McClean \& Millard [38] using relative weightings for each phase based on the then current data for geriatric patients in the UK. These relative weightings are 15, 10, 8 and 6 for hospital phases acute, treatment, rehabilitative and longstay respectively and 5, 4 and 0.5 for community phases dependent, convalescent and recovered respectively [38]. Also McClean \& Millard [38] estimated average daily cost of the care in acute phase as $£ 150$. Here we will consider 2 cases, one with a fixed number of admissions each day and the other with a variable number of admissions each day.

\subsubsection{A fixed number of admissions}

For a new care system [9] with one admission each day (fixed), the expected number of patients in different phases (after a given number of days) is presented in Table 1. Here the average age of patients at the time of admission is 80 years and the initial year of the care system is 1976 . From Table 1, we can also calculate the expected number of patients in any phase or expected cost with any (fixed) number of admissions per day. If the number of admissions per day are $a$ then we need to multiply the corresponding entry in Table 1 by $a$. For example, if in a new hospital, each day on average 100 new patients are admitted, then after 1,000 days, the expected numbers of patients in the hospital and community are estimated as 5051 and 39981 respectively and the expected total daily cost of care is estimated as $£ 1,165,560$. Similarly after 2,000 days, the expected numbers of patients in the hospital and community are estimated as 6420 and 57785 respectively and the expected total daily cost of care is estimated as $£ 1,253,450$.

\subsubsection{A variable number of admissions}

Fiems et al. [13] describe how we can calculate the expected number of patients in different phases, after a given number of days, with a variable rate of admissions per day. Again from (13) we here obtain:

$\mathbf{s}_{k}=\mathbf{x}+r^{*} \mathbf{w}$

where the vector $\mathbf{x}$ represents the expected number of patients in different phases, after a given number of days, for a new care system with one admission per day and $r$ is the rate of change in the admission rate. The vector $\mathbf{w}$ is the expected change in the patients' distribution due to unit (1 patient per day) change in admission rate. However, it is not necessary to start the admission schedule with one

Table 1 Estimated number of patients and cost (new system with 1 admission/ day)

\begin{tabular}{|c|c|c|c|c|c|c|c|c|c|}
\hline Days & Phase1 & Phase2 & Phase3 & Phase4 & Phase 5 & Phase6 & Phase 7 & Phase8 & Total Daily Cost(In $£)$ \\
\hline 100 & 11.07 & 8.95 & 10.16 & 0.27 & 11.16 & 31.65 & 2.85 & 23.89 & $5,223.0$ \\
\hline 200 & 11.97 & 9.67 & 14.29 & 0.88 & 12.50 & 70.13 & 16.28 & 64.27 & $7,471.3$ \\
\hline 300 & 12.60 & 10.16 & 15.94 & 1.50 & 12.95 & 95.47 & 38.94 & 112.44 & $8,931.8$ \\
\hline 400 & 13.04 & 10.50 & 16.94 & 2.06 & 13.10 & 110.38 & 68.06 & 165.92 & $9,895.4$ \\
\hline 500 & 13.36 & 10.74 & 17.70 & 2.55 & 13.09 & 118.00 & 101.21 & 223.35 & $10,527.1$ \\
\hline 600 & 13.60 & 10.93 & 18.32 & 2.97 & 12.99 & 120.78 & 136.51 & 283.90 & $10,940.3$ \\
\hline 700 & 13.81 & 11.08 & 18.87 & 3.34 & 12.84 & 120.51 & 172.51 & 347.04 & $11,214.3$ \\
\hline 800 & 13.99 & 11.22 & 19.39 & 3.66 & 12.66 & 118.41 & 208.18 & 412.47 & $11,403.4$ \\
\hline 900 & 14.18 & 11.36 & 19.91 & 3.94 & 12.49 & 115.29 & 242.78 & 480.04 & $11,543.0$ \\
\hline 1,000 & 14.38 & 11.51 & 20.43 & 4.19 & 12.32 & 111.66 & 275.83 & 549.68 & $11,655.6$ \\
\hline 1,100 & 14.59 & 11.66 & 20.98 & 4.42 & 12.15 & 107.82 & 307.00 & 621.37 & $11,754.1$ \\
\hline 1,200 & 14.82 & 11.83 & 21.55 & 4.62 & 12.00 & 103.93 & 336.12 & 695.12 & $11,846.0$ \\
\hline 1,300 & 15.07 & 12.01 & 22.16 & 4.80 & 11.86 & 100.08 & 363.08 & 770.93 & $11,934.9$ \\
\hline 1,400 & 15.33 & 12.21 & 22.80 & 4.99 & 11.74 & 96.32 & 387.82 & 848.80 & $12,022.4$ \\
\hline 1,500 & 15.61 & 12.41 & 23.47 & 5.16 & 11.62 & 92.64 & 410.34 & 928.74 & $12,109.1$ \\
\hline 1,600 & 15.91 & 12.63 & 24.18 & 5.32 & 11.51 & 89.07 & 430.66 & $1,010.71$ & $12,195.2$ \\
\hline 1,700 & 16.22 & 12.86 & 24.92 & 5.49 & 11.41 & 85.59 & 448.82 & $1,094.68$ & $12,280.8$ \\
\hline 1,800 & 16.55 & 13.10 & 25.70 & 5.66 & 11.31 & 82.21 & 464.88 & $1,180.59$ & $12,365.8$ \\
\hline 1,900 & 16.89 & 13.35 & 26.51 & 5.82 & 11.22 & 78.92 & 478.91 & $1,268.38$ & $12,450.3$ \\
\hline 2,000 & 17.24 & 13.61 & 27.36 & 5.99 & 11.14 & 75.72 & 490.99 & $1,357.96$ & $12,534.5$ \\
\hline
\end{tabular}


Table 2 Estimated values of change multipliers for different phases (new system with 1 admission/ day)

\begin{tabular}{|c|c|c|c|c|c|c|c|c|c|}
\hline Days & Phase1 & Phase2 & Phase3 & phase4 & phase 5 & Phase6 & phase7 & Phase8 & Total daily cost (in $£$ ) \\
\hline 100 & 167.3 & 210.4 & 516.6 & 18.4 & 417.1 & $1,975.9$ & 210.2 & $1,434.1$ & 189,509 \\
\hline 200 & 310.4 & 321.8 & $1,084.7$ & 110.1 & 636.1 & $7,582.6$ & $2,262.6$ & $7,591.6$ & 518,548 \\
\hline 300 & 484.2 & 455.4 & $1,463.7$ & 263.2 & 817.0 & $13,819.4$ & $7,770.0$ & $19,777.1$ & 883,538 \\
\hline 400 & 665.4 & 594.2 & $1,777.9$ & 455.7 & 978.6 & $19,163.7$ & $17,421.6$ & $38,742.9$ & $1,231,388$ \\
\hline 500 & 845.8 & 731.4 & $2,069.0$ & 671.6 & $1,123.0$ & $23,077.0$ & $31,232.7$ & $64,999.4$ & $1,541,227$ \\
\hline 600 & $1,026.9$ & 868.2 & $2,351.0$ & 899.2 & $1,254.4$ & $25,572.0$ & $48,793.5$ & $98,934.8$ & $1,812,459$ \\
\hline 700 & $1,215.0$ & $1,009.1$ & $2,634.8$ & $1,130.1$ & $1,379.7$ & $26,921.5$ & $69,466.6$ & $140,893.1$ & $2,054,930$ \\
\hline 800 & $1,417.2$ & $1,159.9$ & $2,932.3$ & $1,359.2$ & $1,505.8$ & $27,477.1$ & $92,528.9$ & $191,219.5$ & $2,281,731$ \\
\hline 900 & $1,640.1$ & $1,325.7$ & $3,255.0$ & $1,583.3$ & $1,638.4$ & $27,564.7$ & $117,264.4$ & $250,278.3$ & $2,504,815$ \\
\hline 1,000 & $1,888.2$ & $1,510.2$ & $3,612.6$ & $1,801.3$ & $1,780.8$ & $27,436.4$ & $143,015.9$ & $318,454.7$ & $2,732,909$ \\
\hline 1,100 & $2,164.2$ & $1,715.4$ & $4,011.9$ & $2,013.0$ & $1,934.6$ & $27,259.1$ & $169,206.2$ & $396,145.7$ & $2,971,016$ \\
\hline 1,200 & $2,468.7$ & $1,941.9$ & $4,457.2$ & $2,219.2$ & $2,099.1$ & $27,124.0$ & $195,340.7$ & $483,749.1$ & $3,220,849$ \\
\hline 1,300 & $2,800.9$ & $2,189.4$ & $4,950.4$ & $2,421.2$ & $2,272.9$ & $27,066.0$ & $220,998.7$ & $581,650.5$ & $3,481,661$ \\
\hline 1,400 & $3,159.0$ & $2,456.4$ & $5,491.5$ & $2,620.1$ & $2,453.7$ & $27,083.2$ & $245,822.4$ & $690,213.8$ & $3,751,133$ \\
\hline 1,500 & $3,540.3$ & $2,741.0$ & $6,079.0$ & $2,817.4$ & $2,638.7$ & $27,153.9$ & $269,506.6$ & $809,773.2$ & $4,026,123$ \\
\hline 1,600 & $3,941.8$ & $3,041.0$ & $6,710.4$ & $3,014.2$ & $2,825.2$ & $27,248.3$ & $291,791.6$ & $940,627.5$ & $4,303,206$ \\
\hline 1,700 & $4,360.3$ & $3,353.9$ & $7,382.8$ & $3,211.8$ & $3,010.6$ & $27,336.0$ & $312,458.2$ & $1,083,036.5$ & $4,579,022$ \\
\hline 1,800 & $4,792.2$ & $3,677.1$ & $8,092.5$ & $3,411.0$ & $3,192.4$ & $27,390.4$ & $331,326.2$ & $1,237,218.1$ & $4,850,474$ \\
\hline 1,900 & $5,234.3$ & $4,008.0$ & $8,835.7$ & $3,612.7$ & $3,368.4$ & $27,389.5$ & $348,253.7$ & $1,403,347.7$ & $5,114,825$ \\
\hline 2,000 & $5,682.8$ & $4,343.9$ & $9,608.4$ & $3,817.4$ & $3,536.7$ & $27,317.5$ & $363,136.8$ & $1,581,556.5$ & $5,369,748$ \\
\hline
\end{tabular}

admission per day as we can have multiple admissions per day. Therefore, we can generalize (13) to:

$\mathbf{s}_{k}=a^{*}\left(\mathbf{x}+r^{*} \mathbf{w}\right)$

where $a$ represents the initial number of admissions per day.

Table 2 presents the value of the vector $\mathbf{w}$ for a number of time points in the future. As $a$ and $r$ are scalars, we can calculate the estimated number of patients in each phase as a linear combination of $\mathbf{x}$ and $\mathbf{w}$ and similarly we can calculate the cost. For example, with initial rate of admission 100 patients per day and this rate increasing by 1 patient per day $(r=0.01)$, the expected total daily cost of care after 500 days is estimated as $£ 2,593,937$ (100* $(10527.1+0.01 * 1541227))$ and after 1,000 days it is estimated as $£ 3,898,469(100 *(11655.6+0.01 * 2732909))$.

\subsection{Resource constraints}

\subsubsection{A new care system}

If resource availability at a given time in the future is a constraint it is necessary to schedule admissions in advance such that at a given time in the future the number of patients requiring care resources should be equal to the amount of resources available. In Table 3, we have provided a few examples of resource constraints: the total daily cost limit and the number of beds available in the hospital (summing all beds available in all hospital phases acute, treatment, rehabilitative and long-stay) at a given time in the future. We have also calculated the expected number of admissions per day (assuming a

Table 3 Estimated number of admissions per day according to budget or beds availability

\begin{tabular}{|c|c|c|c|c|c|c|}
\hline \multirow[t]{2}{*}{ Day } & \multicolumn{3}{|c|}{$\begin{array}{l}\text { Estimating the number of admissions per day if the constraint is the } \\
\text { daily cost }\end{array}$} & \multicolumn{3}{|c|}{$\begin{array}{l}\text { Estimating the number of admissions per day if the constraint is the } \\
\text { beds availability }\end{array}$} \\
\hline & $\begin{array}{l}\text { Daily cost } \\
\text { limit (in } £ \text { ) }\end{array}$ & $\begin{array}{l}\text { Number of beds } \\
\text { available in the } \\
\text { hospital }\end{array}$ & $\begin{array}{l}\text { Average } \\
\text { admissions } \\
\text { per day }\end{array}$ & $\begin{array}{l}\text { Number of } \\
\text { beds available in } \\
\text { the hospital }\end{array}$ & $\begin{array}{l}\text { Daily cost } \\
\text { (in } £ \text { ) }\end{array}$ & $\begin{array}{l}\text { Average } \\
\text { admissions } \\
\text { per day }\end{array}$ \\
\hline 500 & 800,000 & 3,370 & 76 & 6,000 & 628,690 & 135 \\
\hline 1,000 & 400,000 & 1,734 & 34 & 8,000 & 822,597 & 158 \\
\hline 1,500 & 500,000 & 2,340 & 41 & 4,500 & 458,330 & 79 \\
\hline 2,000 & 750,000 & 3,841 & 60 & 1,700 & 171,991 & 26 \\
\hline
\end{tabular}


fixed number of admissions each day) allowed to meet these constraints.

With a variable number of admissions per day we can have a number of different expected admission schedules to satisfy the same constraints depending on the expected initial rate of admissions $(a)$ and expected rate of change in admission rate $(r)$. For such a care system, usually these decisions are made concerning the resource availability at some time points in the future. We can use (15)-(17) to calculate the expected initial rate of admissions to satisfy the resource constraint for the first given time point in the future. By using (20), (21), (24) and (25) we can then calculate the expected rate of change in admission rate $(r)$ to satisfy constraints relating to two given time points. In Table 4 we have provided some examples of resource constraints for total daily cost constrained at two time points in the future. We have calculated the expected value of $r$ to satisfy these constraints.

\subsubsection{A pre-existing care system}

For a pre-existing care system, first we allocate the care resources to the expected remaining patients from the patients already in the system at time $t=0$ (or today/ present time). Then we allocate the remaining resources to the expected new patients in the same manner as in the case of a new care system discussed above. Using (4), we can compute the expected number of remaining patients in different phases at a given time in the future $\left(t_{\text {given }}\right)$ from patients already in the system at time $t=0$. Daily care cost for such patients can be computed using (8). Again from (4) we obtain:

$\mathbf{s}_{t_{\text {given }}}=\mathbf{s}_{0} * \prod_{i=1}^{t_{\text {given }}} \mathbf{P}^{(i)}=\mathbf{s}_{0} * \mathbf{H}_{t_{\text {given }}}$

where

$\mathbf{H}_{t_{\text {given }}}=\prod_{i=1}^{t_{\text {given }}} \mathbf{P}^{(i)}$

and $\mathbf{H}_{t_{\text {given }}}=\left\{h_{i j}\right\}$ is the matrix of the expected number of patients in phase $j$ at time $t_{\text {given }}$ if there is one patient in phase $i$ at time $t=0$. A typical value of $\mathbf{H}_{t_{\text {given }}}$ for $t_{\text {given }}=$ 1,000 days is:

$$
\mathbf{H}_{t_{\text {given }}=1000}=\left\{\begin{array}{rrrrrrrr}
0.0022 & 0.0017 & 0.0029 & 0.0020 & 0.0017 & 0.0182 & 0.2253 & 0.7460 \\
0.0024 & 0.0018 & 0.0032 & 0.0024 & 0.0019 & 0.0197 & 0.2437 & 0.7251 \\
0.0018 & 0.0013 & 0.0023 & 0.0050 & 0.0014 & 0.0149 & 0.1789 & 0.7943 \\
0.0006 & 0.0005 & 0.0008 & 0.1107 & 0.0009 & 0.0095 & 0.0430 & 0.8341 \\
0.0030 & 0.0023 & 0.0040 & 0.0014 & 0.0023 & 0.0245 & 0.3079 & 0.6547 \\
0.0032 & 0.0024 & 0.0043 & 0.0013 & 0.0025 & 0.0262 & 0.3319 & 0.6282 \\
0.0044 & 0.0033 & 0.0058 & 0.0012 & 0.0034 & 0.0324 & 0.4664 & 0.4831 \\
0 & 0 & 0 & 0 & 0 & 0 & 0 & 1.0000
\end{array}\right\}
$$

and a typical value of $\mathbf{H}_{t_{\text {given }}}$ for $t_{\text {given }}=2,000$ days is:

$$
\mathbf{H}_{t_{\text {given }}=2000}=\left\{\begin{array}{llllllll}
0.0013 & 0.0009 & 0.0018 & 0.0006 & 0.0007 & 0.0050 & 0.0829 & 0.9069 \\
0.0014 & 0.0010 & 0.0019 & 0.0006 & 0.0008 & 0.0054 & 0.0897 & 0.8992 \\
0.0010 & 0.0008 & 0.0014 & 0.0008 & 0.0006 & 0.0040 & 0.0662 & 0.9253 \\
0.0004 & 0.0003 & 0.0005 & 0.0123 & 0.0003 & 0.0018 & 0.0242 & 0.9603 \\
0.0017 & 0.0013 & 0.0024 & 0.0006 & 0.0010 & 0.0068 & 0.1131 & 0.8731 \\
0.0018 & 0.0014 & 0.0026 & 0.0006 & 0.0010 & 0.0073 & 0.1219 & 0.8633 \\
0.0026 & 0.0019 & 0.0037 & 0.0008 & 0.0014 & 0.0102 & 0.1699 & 0.8096 \\
0 & 0 & 0 & 0 & 0 & 0 & 0 & 1.0000
\end{array}\right\}
$$

We can estimate the expected resource requirements for such a care system by using (33) and (34). For example if there are no new admissions to a care system with initially only 500 patients in the acute phase (and no patient in any other phase) then the expected number of patients left in care system after 2,000 days will be $0.65,0.45,0.9,0.3$, $0.35,0.25$ and 41.45 patients respectively in the acute phase, treatment phase, rehabilitative phase, long-stay 
Table 4 Estimated rate of change in admission rate (r) and estimated number of admissions per day (a) to satisfy given budget constraints at two time points in the future

\begin{tabular}{lccccc}
\hline$t_{1}$ in days & $C\left(t_{1}\right)$ in $£$ & $t_{2}$ in days & $\begin{array}{l}C\left(t_{2}\right) \\
\text { in } £\end{array}$ & $\begin{array}{l}A_{\text {req }}=a \text { (Estimated initial } \\
\text { admission rate) }\end{array}$ & $\begin{array}{l}r \text { (estimated rate of change } \\
\text { in admission rate) }\end{array}$ \\
\hline 500 & 800,000 & 1,000 & 800,000 & 88.23 & -0.00095 \\
500 & 800,000 & 1,000 & 120,240 & 30.83 & 0.01 \\
1,000 & 400,000 & 1,500 & 500,000 & 17.64 & 0.04 \\
1,000 & 500,000 & 1,200 & 400,000 & 100 & -0.0024354 \\
1,500 & 500,000 & 2,000 & 610,000 & 15.73 & 0.049 \\
1,600 & 200,000 & 2,000 & 200,000 & 18.47 & -0.0032 \\
\hline
\end{tabular}

phase, dependent phase, convalescent phase and recovered phase.

We can use (28) and (29) to estimate the expected admission rate to satisfy the given bed availability constraints and the expected cost of care constraints respectively. For example if a care system with initially 100 patients in each phase has an expansion plan to double the expected total daily cost of care after 1,000 days, then

$\widetilde{\mathbf{s}}_{0}=\left\{\begin{array}{llllllll}100 & 100 & 100 & 100 & 100 & 100 & 100 & 0\end{array}\right\}$

and

$\widetilde{\Omega}_{0}=£ 48500$.

The constraint is

$\mathrm{C}\left(t_{\text {given }}=1000\right)=2 * \widetilde{\Omega}_{0}=£ 97000$.

From (33) and (34) we obtain $\widetilde{\mathbf{s}}_{t_{\text {given }}=1000}=\widetilde{\mathbf{s}}_{0} * \mathbf{H}_{t_{\text {grien }}=1000}=$ $\left\{\begin{array}{llllllll}1.76 ; & 1.33 & 2.33 & 12.4 & 1.41 & 14.54 & 179.71 & 486.55\end{array}\right\}$, and $\widetilde{\Omega}_{t_{\text {griven }}=1000}=£ 2878$.05. From (29), the mean rate of admissions allowed (with a fixed number of admissions each day) will be

$A_{\text {req }}=\frac{C\left(t_{\text {given }}=1000\right)-\widetilde{\Omega}_{t_{\text {given }}=1000}}{\Omega_{t_{\text {given }}=1000}}$ per day $=\frac{97000-2878.05}{\Omega_{t_{\text {given }}=1000}}$.

From Table 1, $\Omega_{t_{\text {given }}=1000}=£ 11655.60$

Therefore, $A_{\text {req }}=8.0753$ patients/day.

For a care system with variable number of admissions each day, if the initial rate of admission $(a)$ is 10 patients/ day, then using (31) the rate of change in admission rate $(r)$ can be estimated as follows:

$r=\frac{C\left(t_{\mathrm{given}}=1000\right)-\widetilde{\Omega}_{t_{\mathrm{given}=1000}}-a^{*}\left(\mathbf{x}^{*} \mathbf{c}\right)}{a^{*}\left(\mathbf{w}^{*} \mathbf{c}\right)}$.

Putting the values of $\left(\mathbf{x}^{*} \mathbf{c}\right)$ and $\left(\mathbf{w}^{*} \mathbf{c}\right)$ from the Tables 1 and 2 respectively we get

$r=\frac{97000-2878.05-(10 * 11655.6)}{10 * 2732909}=-0.000821$.
If the same care system has a contraction plan to half the expected total daily cost of care after 1,000 days, then the constraint is

$\mathrm{C}\left(t_{\text {given }}=1000\right)=\frac{\widetilde{\Omega}_{0}}{2}=£ 24250$.

From (29), the mean rate of admissions allowed (with a fixed number of admissions each day) will be

$A_{\text {req }}=\frac{C\left(t_{\text {given }}=1000\right)-\widetilde{\Omega}_{t_{\text {given }}=1000}}{\Omega_{t_{\text {given }}=1000}}$ per day $=\frac{24250-2878.05}{\Omega_{t_{\text {given }}=1000}}$.

From Table 1, $(\mathbf{x} * \mathbf{c}) \Omega_{t_{\text {given }}=1000}=£ 11655.60$

Therefore, $A_{\text {req }}=1.8336$ patients/day.

For a care system with variable number of admissions each day, if the initial (or present) rate of admission $(a)$ is 10 patients/day, then using (31) the rate of change in admission rate $(r)$ can be estimated as follows:

$r=\frac{C\left(t_{\text {given }}=1000\right)-\widetilde{\Omega}_{t_{\text {given }=1000}}-a^{*}\left(\mathbf{x}^{*} \mathbf{c}\right)}{a^{*}\left(\mathbf{w}^{*} \mathbf{c}\right)}$.

Putting the values of and $\left(\mathbf{w}^{*} \mathbf{c}\right)$ from Tables 1 and 2 respectively we get

$r=\frac{24250-2878.05-(10 * 11655.6)}{10 * 2732909}=-0.003483$.

If the bed availability is a constraint, then we can use (28) to estimate the mean rate of admissions allowed for a care system with a fixed number of admissions each day and (30) to estimate the rate of change in admission rate $(r)$ for a care system with variable number of admissions each day.

\section{Discussion}

In our model, we assume that there is always a waiting list of patients who can be admitted to the first phase of the care system whenever there is a bed available. This assumption is realistic in many practical situations. However, as the transition probabilities are estimated separately 
for each patient in the care system, the model can be modified for resource requirements planning in other scenarios where patients arrive arbitrarily and admissions can be modeled as a Poisson stream. Another limitation of our model is that it is based largely on expected values. The model can therefore be used to predict the expected number of patients in each phase and expected daily cost of care. However, these expected numbers represent long term averages, but do not reflect variability. We are currently working on enhancing our model to estimate the variability of these averages and to examine its effects. Such variability and its effects can also be estimated using a stochastic simulation models (such as a discrete event simulation).

An obvious application of our model is to facilitate decision making of health and social care planners, managers and policy makers for resource planning and budget allocation, especially for the elderly care. However, this model can be customized for other care systems as well. Our model equips care planners, managers and policy makers with a tool for resource planning, budget allocation and strategic decision making for care management and service improvement. In a community based setting, they can use our model with demographic data and regional health statistics data, to allocate budget for health and social care for the population of their region. Bed availability, optimum utilization of the allocated budget, minimization of waiting time and the size of patient queues can thus be assured Individual resource requirements for hospital care and community care can also be estimated. Another application of the tool is in health insurance where an insurance company might use the model to forecast resource requirements and then decide the optimum premium for the patient's health insurance policy.

Our model can also be used for better informed budget allocation and resource planning decisions evaluating the combined cost measure of the dead-alive trade off, quality of life and economic costs [39]. This can be achieved by using our model coupled with a partially observable Markov decision process based on, for example, the patient management model proposed by [39] to select the care pathway which optimizes the combined cost for the integrated care system. This will also facilitate cost effectiveness analysis (CEA) [40-42] and cost-utility analysis (CUA) [40, 43] of different admission scheduling policies and resource management strategies. For example we might decide if it is costeffective to discharge (from a hospital phase) a patient to a care home or nursing home or his/her own home, with a care package such as is described in [44]. We can estimate the resource availability and schedule the admissions accordingly. Quality of life can be measured in terms of quality adjusted life years gained (QALY) [45], disability adjusted life years (DALYs) [45], life years gained (LYs gained) [45] or Sen's capability approach [46, 47]. It can also be measured for individual patients in the care system facilitating combined cost effectiveness analysis for the whole the system as in the model the transition probabilities are estimated separately for each patient.

To understand the effect of change in the various input parameters we carried out the sensitivity analysis of various input parameters (see Appendix 1 for the mathematical details). The results of this analysis can be summarized as follows:

- The mean number of patients in each phase on a given day in the future increases exponentially with the change in a transition rate (a parameter of transition matrix Q) (see Eqs. 35 and 36, Appendix 1).

- The mean number of patients in each phase on a given day in the future increases linearly with the change in the initial (or fixed) admission rate (see Eq. 37, Appendix 1).

- The mean number of patients in each phase on a given day in the future increases linearly with the change in the rate of change in the admission rate (see Eq. 38, Appendix 1).

- Changes in the expected total daily cost on a given day in the future increases linearly with changes in daily cost of care in different phases (and similarly with the change in the relative weighting for a phase for indicative cost) (see Eq. 39, Appendix 1).

- If the beds availability on a given day in the future is a constraint, then the expected number of admissions allowed each day decreases exponentially with the change in a transition rate (see Eqs. 40 and 41, Appendix 1).

- If the daily cost of care on a given day in the future is a constraint, then the expected number of admissions allowed each day decreases approximately linearly with changes in daily cost of care in different phases (and similarly with the change in the relative weighting for a phase for indicative cost) (see Eqs. 42 and 43, Appendix 1).

Our model is very efficient in terms of algorithm complexity. Implementation of the algorithm discussed in the last section for resource requirements forecasting and/or admission scheduling would require matrix multiplications for each new admission and for each day throughout the duration we wish to forecast the resource requirement. Also the number of admissions is equal to the time horizon of the resource requirement. Therefore the time complexity of the algorithm is $\mathrm{O}\left(k^{2}\right)$ where $k$ is the number of days ahead for which the resource requirement requires to be forecasted. Also, we require to store the previous value of matrix $\mathbf{s}_{k}$ for each iteration. Therefore the space complexity of the algorithm is $\mathrm{O}(n)$. 


\section{Conclusion}

We have demonstrated how a discrete time nonhomogeneous Markov models can be effectively used in more sophisticated admission scheduling and resource requirement forecasting and allocation. We allow both fixed and variable rate of admissions to satisfy the demand or resource constraints. This can be a very useful tool for care managers and policy makers so as to facilitate strategic decision making for care management and service improvement. We are currently working to develop our approach as a decision (what-if analysis) tool.

Acknowledgements The authors acknowledge support for this work from the Engineering and Physical Sciences Research Council funded RIGHT and MATCH projects (Grant References EP/E019900/1 and GR/S29874/01). Any views or opinions presented herein are those of the authors and do not necessarily represent those of RIGHT or $\mathrm{MATCH}$, their associates or their sponsors

\section{Appendix 1: Equations for sensitivity analysis}

The mean number of patients in each phase after $k$ days

\section{The change in the transition rates}

From Eq. 4, the mean number of patients in each phase after $k$ days is given by:

$\mathbf{s}_{k}=\mathbf{s}_{0} * \prod_{i=1}^{k} \mathbf{P}^{(i)}$

where $\mathbf{P}^{(i)}$ represents the value of transition matrix $\mathbf{P}$ on day $i$ which is defined in Eq. 2 as follows

$\mathbf{s}_{k}=\mathbf{s}_{0} * \prod_{i=1}^{k}\left(\exp \left(\mathbf{Q}^{(i)}\right)\right)$

Therefore,

$$
\begin{aligned}
\Rightarrow \mathbf{s}_{k}+\Delta_{\mathbf{Q}} \mathbf{s}_{k} & =\mathbf{s}_{0} * \prod_{i=1}^{k}\left(\exp \left(\mathbf{Q}^{(i)}+\Delta \mathbf{Q}^{(i)}\right)\right) \\
& =\mathbf{s}_{0} * \prod_{i=1}^{k}\left(\exp \left(\mathbf{Q}^{(i)}\right) * \exp \left(\Delta \mathbf{Q}^{(i)}\right)\right) \\
\Rightarrow \mathbf{s}_{k}+\Delta_{\mathbf{Q}} \mathbf{s}_{k} & =\mathbf{s}_{0} * \prod_{i=1}^{k}\left(\exp \left(\mathbf{Q}^{(i)}\right)\right) * \prod_{i=1}^{k}\left(\exp \left(\Delta \mathbf{Q}^{(i)}\right)\right) \\
\Rightarrow \mathbf{s}_{k}+\Delta_{\mathbf{Q}} \mathbf{s}_{k} & =\mathbf{s}_{k} * \prod_{i=1}^{k}\left(\exp \left(\Delta \mathbf{Q}^{(i)}\right)\right) .
\end{aligned}
$$

Here $\Delta \mathbf{Q}$ represents the change in the transition matrix $\mathbf{Q}$ defined by Eq. 1. There might be equal changes in all transition rates which represents a global shift in transition rates, or there might be just change in one transition rate such as a change in the value of $\mu_{l}$. If the latter is the case then,

$\exp (\Delta \mathbf{Q})=\exp \left(\Delta \mu_{l}\right)$

Therefore

$\mathbf{s}_{k}+\Delta_{\mathbf{Q}} \mathbf{s}_{k}=\mathbf{s}_{k} * \prod_{i=1}^{k}\left(\exp \left(\Delta \mu_{l}^{(i)}\right)\right)$.

The change in the initial number of admissions per day

If the initial number of admissions per day is $a$ with a variable rate of admissions per day, then from Eq. 30, the expected number of patients in different phases after a given number of days,

$\mathbf{s}_{k}=a^{*}\left(\mathbf{x}+r^{*} \mathbf{w}\right)$

$\mathbf{s}_{k}+\Delta_{a} \mathbf{s}_{k}=(a+\Delta a)^{*}\left(\mathbf{x}+r^{*} \mathbf{w}\right)$

$\mathbf{s}_{k}+\Delta_{a} \mathbf{s}_{k}=\mathbf{s}_{k}+\Delta a^{*}\left(x+r^{*} \mathbf{w}\right) \quad \frac{\Delta_{a} \mathbf{s}_{k}}{\mathbf{s}_{k}}=\frac{\Delta a}{a}$

The change in the rate of change in the admission rate

Again from Eq. 30, for calculating the sensitivity of $r$ the rate of change in the admission rate.

$\mathbf{s}_{k}=a^{*}\left(\mathbf{x}+r^{*} \mathbf{w}\right)$

$\mathbf{s}_{k}+\Delta_{r} \mathbf{s}_{k}=a^{*}(\mathbf{x}+(r+\Delta r) * \mathbf{w})$

$\mathbf{s}_{k}+\Delta_{r} \mathbf{s}_{k}=a^{*}\left(\mathbf{x}+(r+\Delta r)^{*} \mathbf{w}\right)=a^{*}\left(\mathbf{x}+r^{*} \mathbf{w}\right)+a^{*} \Delta r^{*} \mathbf{w}$

$\mathbf{s}_{k}+\Delta_{r} \mathbf{s}_{k}=\mathbf{s}_{k}+a^{*} \Delta r^{*} \mathbf{w}$

$\frac{\Delta_{r} \mathbf{s}_{k}}{\mathbf{s}_{k}}=\frac{\Delta r^{*} \mathbf{w}}{\left(\mathbf{x}+r^{*} \mathbf{w}\right)}$

The expected total daily cost on dayk

The change in cost matrix

From Eq. 8, the expected total daily cost on day $k$ is given as follows

$\Omega_{k}=\mathbf{s}_{k} * \mathbf{c}$

$\Rightarrow \Omega_{k}+\Delta_{\mathbf{c}} \Omega_{k}=\mathbf{s}_{k} *(\mathbf{c}+\Delta \mathbf{c})$

$\Rightarrow \Omega_{k}+\Delta_{\mathbf{c}} \Omega_{k}=\Omega_{k}+\mathbf{s}_{k} * \Delta \mathbf{c}$

$\Rightarrow \frac{\Delta_{\mathbf{c}} \Omega_{k}}{\Omega_{k}}=\frac{\Delta \mathbf{c}}{\mathbf{c}}$.

Here $\Delta \mathbf{c}$ represents the change in cost matrix $\mathbf{c}$ defined in Section 3.1. Section 4.1 defines $\mathbf{c}$ in the form of relative 
weightings. In such a case $\Delta \mathbf{c}$ represents the change in one or more weightings.

The expected number of admissions allowed each day

\section{The change in the transition rates}

From Eq. 15 the expected number of admissions allowed each day can be calculated as:

$A_{\text {req }}=\frac{B\left(t_{\text {given }}\right)}{\eta_{t_{\text {given }}}}$ per day

where $\eta_{t_{\text {given }}}$ is defined in the Eq. 5

$\eta_{t_{\text {given }}}=\sum_{i=1}^{n+m} s_{t_{\text {given }, i}}=\mathbf{s}_{t_{\text {given }}} *(\mathbf{h}+\mathbf{e})$

Therefore

$$
\begin{gathered}
A_{\text {req }}+\Delta_{\mathbf{Q}} A_{\text {req }}=\frac{B\left(t_{\text {given }}\right)}{\eta_{t_{\text {given }}} * \prod_{i=1}^{t_{\text {given }}}\left(\exp \left(\Delta \mathbf{Q}^{(i)}\right)\right)} \text { per day } \\
\Rightarrow A_{\text {req }}+\Delta_{\mathbf{Q}} A_{\text {req }}=\frac{A_{\text {req }}}{\prod_{i=1}^{t_{\text {given }}}\left(\exp \left(\Delta \mathbf{Q}^{(i)}\right)\right)} \text { per day }
\end{gathered}
$$

If $\Delta \mathbf{Q}$ represents the change in only one transition rate such as $\mu_{l}$ then

$$
A_{\text {req }}+\Delta_{\mathbf{Q}} A_{\text {req }}=\frac{A_{\text {req }}}{\prod_{i=1}^{t_{\text {given }}}\left(\exp \left(\Delta \mu_{l}^{(i)}\right)\right)} \text { per day }
$$

\section{The change in cost matrix}

If the total daily cost of care is a constraint then the expected number of admissions is calculated in Eq. 17:

$A_{\text {req }}=\frac{C\left(t_{\text {given }}\right)}{\Omega_{t_{\text {given }}}}$ per day

$\Omega_{t_{\text {given }}}$ is the expected total daily cost at time $t_{\text {given }}$ and is defined in (8). Therefore

$A_{\text {req }}+\Delta_{\mathbf{c}} A_{\text {req }}=\frac{C\left(t_{\text {given }}\right)}{\Omega_{t_{\text {given }}}+\mathbf{s}_{t_{\text {given }}} * \Delta \mathbf{c}}$ per day

$A_{\text {req }}+\Delta_{\mathbf{c}} A_{\text {req }}=\frac{A_{\text {req }}}{1+(\Delta \mathbf{c} / \mathbf{c})}$ per day

$\frac{\Delta_{\mathbf{c}} A_{\text {req }}}{A_{\text {req }}}=\frac{-\Delta \mathbf{c} / \mathbf{c}}{1+(\Delta \mathbf{c} / \mathbf{c})}$ per day $=\frac{-1}{1+(\mathbf{c} / \Delta \mathbf{c})}$ per day
If $\Delta \mathbf{c}<<\mathbf{c}$ then

$\frac{\Delta_{\mathbf{c}} A_{\text {req }}}{A_{\text {req }}} \approx \frac{-\Delta \mathbf{c}}{\mathbf{c}}$ per day

\begin{tabular}{|c|c|}
\hline & The number of hospital phases in the care system. \\
\hline$m$ & The number of community phases in the care system. \\
\hline $\mathbf{Q}$ & $\begin{array}{l}\text { The transition matrix for the absorbing Markov } \\
\text { representing the care system. }\end{array}$ \\
\hline$q_{i j}$ & $\begin{array}{l}\text { The transition rate (next transition is to phase } j \mid \\
\text { currently in phase } i \text { ). }\end{array}$ \\
\hline$\lambda_{i}$ & $\begin{array}{l}\text { The rate of transition from the hospital phase } i \text { to } \\
\text { the hospital phase } i+1 \text {. }\end{array}$ \\
\hline$\mu_{i}$ & $\begin{array}{l}\text { The rate of transition from the hospital phase } i \text { to the } \\
\text { absorbing phase (death). }\end{array}$ \\
\hline$\nu_{i}$ & $\begin{array}{l}\text { The rate of transition from the hospital phase } i \text { to the } \\
\text { first community phase. }\end{array}$ \\
\hline$\alpha_{i}$ & $\begin{array}{l}\text { The rate of transition from the community phase } i \text { to } \\
\text { the community phase } i+1 \text {. }\end{array}$ \\
\hline$\beta_{i}$ & $\begin{array}{l}\text { The rate of transition from the community phase } i \text { to } \\
\text { the absorbing phase (death). }\end{array}$ \\
\hline$\gamma_{i}$ & $\begin{array}{l}\text { The rate of transition from the community phase } i \text { to } \\
\text { the first hospital phase. }\end{array}$ \\
\hline $\mathbf{P}$ & The discrete time transition probability matrix. \\
\hline$p_{i j}$ & $\begin{array}{l}\text { Probability (next transition is to phase } j \mid \text { currently in } \\
\text { phase } i \text { ). }\end{array}$ \\
\hline $\mathbf{P}^{(i)}$ & The value of transition matrix $\mathbf{P}$ on day $i$. \\
\hline $\mathbf{P}_{j}^{(i)}$ & The transition probability matrix for patient $j$ on day $i$. \\
\hline$t$ & Time in days. \\
\hline$t_{i}$ & A date in the future \\
\hline$t_{\text {given }}$ & A given time in the future \\
\hline $\mathbf{s}_{\mathbf{0}}$ & $\begin{array}{l}\text { The vector representing initial values of number of } \\
\text { patients in different phases of the system. }\end{array}$ \\
\hline$s_{0, i}$ & The initial value of number of patients in phase $i$. \\
\hline$\widetilde{\mathbf{s}}_{0}$ & $\begin{array}{l}\text { The vector representing values of number of (pre-existing) } \\
\text { patients in different phases of the system at } t=0 .\end{array}$ \\
\hline$s_{k}$ & The mean number of patients in each phase after $k$ days. \\
\hline$\widetilde{\mathbf{s}}_{\text {given }}$ & $\begin{array}{l}\text { The distribution of the patients at time } t_{\text {given }} \text { without } \\
\text { any new admissions }\end{array}$ \\
\hline$\widetilde{\mathbf{s}}_{t_{\text {given }}}=1000$ & $\begin{array}{l}\text { The distribution of the patients at time } t_{\text {given }}=1,000 \\
\text { days without any new admissions }\end{array}$ \\
\hline c & $\begin{array}{l}\text { The cost vector representing the daily cost of care in } \\
\text { different phases. }\end{array}$ \\
\hline$c$ & daily cost of care in phase $i$. \\
\hline$C\left(t_{\text {given }}\right)$ & The available daily budget (cost) at time $t_{\text {given }}$ \\
\hline$C\left(t_{\text {given }=1,000}\right)$ & $\begin{array}{l}\text { The available daily budget (cost) at time } \\
t_{\text {given }}=1,000 \text { days }\end{array}$ \\
\hline$C\left(t_{i}\right)$ & the total daily expenses (cost) at time $t_{i}$ \\
\hline & Vector of time dependent covariates. \\
\hline
\end{tabular}

\section{Appendix 2}

Table 5 Terms/ parameters used in the paper 


$\begin{array}{ll}\chi_{i} & \text { The } i^{\text {th }} \text { time dependent covariate. } \\ \sigma & \text { A coefficient parameter used in the log-linear function. } \\ \mathbf{B} & \text { A vector used in the log-linear function. } \\ \eta_{k} & \text { The estimated number of patients in the care system on day } \\ & k . \\ \eta_{k}{ }^{h} & \text { The expected number of patients in hospital after } k \text { days. } \\ \eta_{k}{ }^{c} & \text { The expected number of patients in community } \\ & \text { after } k \text { days. } \\ \eta_{t_{i}} & \text { The expected number of patients in the care system } \\ \widetilde{\eta}_{t_{\text {given }}} & \text { at time } t_{i} \\ \eta_{t_{\text {given }}} & \text { The expected number of patients in the care system } \\ \eta_{t_{\text {given }}}^{h} & \text { at time } t_{\text {given }} \text { The expected number of patients in the care system } \\ \mathbf{h} & \text { at time } t_{\text {given }} \\ & \text { The number of patients in hospital at time } t_{\text {given }} \\ \mathbf{e} & \text { A column vector of }(n+m+1) \text { elements with the first } \\ & n \text { elements equal to } 1 \text { and the remaining elements } \\ & \text { equal to } 0 .\end{array}$

$\Omega_{\boldsymbol{k}} \quad$ The expected total daily cost on day $k$.

$\Omega_{t_{\text {given }}} \quad$ The expected total daily cost at time $t_{\text {given }}$

$\Omega_{t_{\text {given }}=1000}$ The expected total daily cost at time $t_{\text {given }}=1,000$ days

$\Omega_{t_{i}} \quad$ the expected total daily cost on day $t_{i}$

$\widetilde{\Omega}_{t_{\text {given }}} \quad$ The expected total daily cost at time $t_{\text {given }}$ without any new admissions

$\widetilde{\Omega}_{t_{\text {given }}=1000} \quad$ The expected total daily cost at time $t_{\text {given }}=1,000$ days (without any new admissions)

$A$

$A_{\text {req }}$

$R$

$\mathbf{x}$

$\mathbf{x}_{i}$

Y

W

$\mathbf{w}_{i}$

$B\left(t_{\text {given }}\right)$
$\mathrm{B}\left(t_{i}\right)$
$\mathbf{H}_{t_{\text {given }}}$

$\mathbf{H}_{t_{\text {given }}}=1000$

$h_{i j}$

$\Delta \mathbf{Q}^{(i)}$

$\Delta \mu_{l}^{(i)}$
$\Delta_{\mathbf{Q}} \mathbf{s}_{k}$

$\Delta a$

$\Delta_{a} \mathbf{s}_{k}$

$\Delta r$

$\Delta_{r} \mathbf{s}_{k}$

$\Delta \mathbf{c}$

$\Delta_{\mathbf{c}} \Omega_{k}$

$\Delta_{\mathbf{Q}} A_{\text {req }}$

$\Delta_{\mathbf{c}} A_{\text {req }}$

The change in the distribution of the patients at day $k$ as a result of the change in transition matrix $\mathbf{Q}$

The change in the initial (or fixed) number of admissions per day

The change in the distribution of the patients at day $k$ as a result of the change in the initial (or fixed) number of admissions per day

The change in the rate of change in the admission rate

The change in the distribution of the patients at day $k$ as a result of the change in the rate of change in the admission rate

The change in cost vector $\mathbf{c}$ (cost of care in different phases)

The change in the total daily cost of care on day $k$ as a result of the change in cost matrix c

The change in the expected number of admissions allowed each day to satisfy a given constraint as a result of the the change in transition matrix $\mathbf{Q}$

The change in the expected number of admissions allowed each day to satisfy a given constraint as a result of the change in cost matrix $\mathbf{c}$

\section{References}

1. Gemmel P, van Dierdonck R (1999) Admission scheduling in acute care hospitals: does the practice fit with the theory? Int $\mathrm{J}$ Oper Prod Manage 19(9):863-878

2. Milsum JH, Turban E, Vertinsky I (1973) Hospital admission systems: their evaluation and management. Manage Sci 19 (6):646-666

3. Shaw B, Marshall AH (2005) A Bayesian approach to modelling inpatient expenditure. Proceedings of the 18th IEEE Symposium on Computer-Based Medical Systems, pp 491-496

4. Buhaug H (2002) Long waiting lists in hospitals. BMJ 324 (7332):252-253

5. Worthington DJ (1987) Queueing models for hospital waiting lists. J Oper Res Soc 38(5):413-422

6. Gupta D, Natarajan MK, Gafni A, Wang L, Shilton D, Holder D, Yusuf S (2007) Capacity planning for cardiac catheterization: a case study. Health Policy (Amsterdam) 82(1):1-11

7. Murray M, Berwick DM (2003) Advanced access: reducing waiting and delays in primary care. J Am Med Assoc 289(8):1035-1040

8. Groot PMA (1993) Decision support for admission planning under multiple resource constraints. Dissertation, Eindhoven University of Technology

9. Worthington DJ (1991) Hospital waiting list management models. J Oper Res Soc 42(10):833-843

10. Gorunescu F, McClean SI, Millard PH (2002) A queuing model for bed-occupancy management and planning of hospitals. J Oper Res Soc 53:19-24

11. Cochran J, Roche K (2007) A queuing-based decision support methodology to estimate hospital inpatient bed demand. J Oper Res Soc 59:1471-1482. doi:10.1057/palgrave.jors.2602499

12. Fomundam S, Herrmann JW (2007) A survey of queuing theory applications in healthcare. ISR technical report, Technical Report 2007-24, College Park (MD): Institute for Systems Research, University of Maryland

13. Fiems D, Koole G, Nain P (2005) Waiting times of scheduled patients in the presence of emergency requests. Available online. http://www.math.vu.nl/ koole/articles/report05a/art.pdf. title of subordinate document. Accessed 12 Aug 2008 
14. Kuzdrall PJ, Kwak NK, Schmitz HH (1981) Simulating space requirements and scheduling policies in a hospital surgical suite. Simulation 36(5):163-171

15. Vassilacopoulos G (1985) A simulation model for bed allocation to hospital inpatient departments. Simulation 45(5):233-241

16. Lehaney B, Hlupic V (1995) Simulation modelling for resource allocation and planning in the health sector. J R Soc Health 115 (6):382-385

17. Fone D, Hollinghurst S, Temple M, Round A, Lester N, Weightman A, Roberts K, Coyle E, Bevan G, Palmer S (2003) Systematic review of the use and value of computer simulation modelling in population health and health care delivery. J Public Health Med 25 (4):325-335

18. Jacobson SH, Hall SN, Swisher James R (2006) Discrete-event simulation of health care systems. In: Patient flow: reducing delay in healthcare delivery. Springer, US, pp 211-252

19. Vissers JMH, Adan IJBF, Dellaert NP (2007) Developing a platform for comparison of hospital admission systems: An illustration. Eur J Oper Res 180(3):1290-1301

20. Williams SV (1983) How many intensive care beds are enough? Crit Care Med 11:412-416

21. Jung AL, Streeter NS (1985) Total population estimate of newborn special-care bed needs. Pediatrics 75:993-996

22. Plati C, Lemonidou C, Priami M, Baltopoulos G, Mantas J (1996) The intensive care units in greater Athens: needs and resources. Intensive Crit Care Nurs 12:340-345

23. Parmanum J, Field D, Rennie J, Steer P (2000) National census of availability of neonatal intensive care. BMJ 321:727-729

24. Lampl C, Klingler D, Deisenhammer E, Hagenbichler E, Neuner L, Pesec B (2001) Hospitalization of patients with neurological disorders and estimation of the need of beds and of the related costs in Austria's non-profit hospitals. Eur J Neurol 8:701-706

25. Nguyena JM, Sixc P, Antoniolib D, Glemaind P, Potele G, Lombrailb P, Le Beuxf P (2005) A simple method to optimize hospital beds capacity. Int J Med Inform 74(1):39-49

26. Mackay M, Lee M (2005) Choice of models for the analysis and forecasting of hospital beds. Health Care Manage Sci 8:221-230

27. Ivatts S, Millard P (2002) Health care modelling-why should we try? Br J Health Care Manag 8(6):218-222

28. Plochg T, Klazinga NS (2002) Community-based integrated care: myth or must? Int J Qual Health Care 14(2):91-101

29. Garg L, McClean SI, Meenan B, Millard PH (2008) Optimal control of patient admissions to satisfy resource restrictions. Proceedings of the 21st IEEE Symposium on Computer-Based Medical Systems, pp 512-517

30. Shonick W (1972) Understanding the nature of the random fluctuations of the hospital daily census: an important health planning tool. Med Care 10(2):118-142
31. McClean SI, Millard PH (1993) Patterns of length of stay after admission in geriatric medicine: an event history approach. Statistician 42(3):263-274

32. Marshall A, Vasilakis C, El-Darzi E (2005) Length of stay-based patient flow models: recent developments and future directions. Health Care Manage Sci 8(3):213-220

33. Faddy MJ, McClean SI (1999) Analysing data on lengths of stay of hospital patients using phase-type distributions. Appl Stoch Models Bus Ind 15(4):311-317

34. Garg L, McClean SI, Meenan BJ, Millard PH (2008) Nonhomogeneous Markov models for sequential pattern mining of healthcare data. IMA J Manag. Math. doi:10.1093/imaman/dpn030

35. Faddy MJ, McClean SI (2005) Markov chain modelling for geriatric patient care. Methods Inf. Med 44(3):369-373

36. Nelder JA, Mead R (1965) A simplex method for function minimization. Comput $\mathrm{J}$ 7:308-313

37. MATLAB, The Language of Technical Computing, Version 7.7.0.471 (R2008b), September 17, 2008, The MathWorks, Inc., Natick, Massachussetts

38. McClean SI, Millard PH (2006) Where to treat the older patient? Can Markov models help us better understand the relationship between hospital and community care? J Oper Res Soc 58 (2):255-261

39. Hauskrecht M, Fraser H (2000) Planning Treatment of ischemic heart disease with partially observable Markov decision processes. Artif Intell Med 18:221-244

40. Stothers L (2007) Cost-Effectiveness Analyses. In: Penson DF, Wei JT (eds) Clinical research methods for surgeons. Humana, Totowa, pp 283-296

41. Weinstein MC, Stason WB (1977) Foundations of costeffectiveness analysis for health and medical practices. $\mathrm{N}$ Engl $\mathrm{J}$ Med 296:716-721

42. Kocher MS, Henley MB (2003) It is money that matters: decision analysis and cost effectiveness analysis. Clin Orthop Relat Res 413:106-116

43. Romangnuolo J, Meier MA (2002) Medical or surgical therapy for erosive reflux esophagitis: cost-utility analysis using a Markov model. Ann Surg 236(2):191-202

44. Rowland DR, Pollock AM (2004) Choice and responsiveness for older people in the "patient centred" NHS. BMJ 328:4-5. doi:10.1136/bmj.328.7430.4

45. Robberstad B (2005) QALYs vs DALYs vs LYs gained: what are the differences, and what difference do they make for health care priority setting? Nor Epidemiol 15(2):183-191

46. Sen A (1993) Capability and well-being. In: Nussbaum M, Sen A (eds) The quality of life. Clarendon, Oxford, pp 30-54

47. Cookson R (2005) QALYs and the capability approach. Health Econ 14:817-829 